\title{
O uso do "photoscreening" no estudo de fatores ambliopigênicos na população infantil
}

\author{
The use of Photoscreening in the study of the amblyopigenics factors in children
}

Pedro Paulo Leite dos Reis ${ }^{(1)}$

Wagner Oto Kawakami ${ }^{(2)}$

Domingos A. C. Mourão ${ }^{(2)}$
Trabalho realizado pelos médicos do HOMG, durante o projeto "Veja Bem Brasil", na cidade de Santa Luzia/MG.

(1) Doutor em Oftalmologia pela UFMG. Coordenador do Serviço de Estrabismo do Hospital de Olhos de Minas Gerais (HOMG)

(2) Oftalmologista e Fellow do Hospital de Olhos de Minas Gerais.

Os autores declaram que não possuem interesse financeiro no desenvolvimento ou marketing do aparelho referido no estudo.

Endereço para correspondência: Rua Santa Catarina, 1300/402. Bairro de Lourdes, Belo Horizonte (MG) CEP 30170-081. e-mail: hospital@holhos.com.br

\begin{tabular}{|c|}
\hline RESUMO \\
\hline $\begin{array}{l}\text { Objetivo: Estudar a precisão do aparelho "MTI Photoscreener" } \\
\text { na triagem de uma população infantil,identificando fatores ambliopigê- } \\
\text { nicos como ametropias, anisometropias, estrabismos e cataratas } \\
\text { congênitas e opacidades dos meios transparentes. } \\
\text { Métodos: Durante o projeto "Veja Bem Brasil“" os autores exami- } \\
\text { naram com uma máquina de "photoscreening" (MTI Photoscree- } \\
\text { ner), } 107 \text { crianças, com idade variando entre } 6 \text { e } 15 \text { anos, comparando } \\
\text { estes resultados com aqueles encontrados em posterior exame } \\
\text { oftalmológico completo. } \\
\text { Resultados: Ao final encontraram } 31 \text { (28,97\%) crianças com algu- } \\
\text { ma patologia oftalmológica e } 76(71,02 \%) \text { dentro da normalidade. As } \\
\text { análises estatísticas da precisão do aparelho revelaram os seguintes } \\
\text { valores: sensibilidade } 90,32 \% \text {; especificidade, } 96,05 \% \text {; valores pre- } \\
\text { ditivos positivos e negativos de } 90,32 \% \text { e } 96,05 \% \text {, respectivamente. } \\
\text { Conclusão: Os resultados acima citados demonstraram a confia- } \\
\text { bilidade do teste de "Photoscreening" na triagem de pacientes em } \\
\text { idade escolar. }\end{array}$ \\
\hline
\end{tabular}

Palavras-chave: Triagem; "Photoscreening" (crianças); Ambliopia.

\section{INTRODUCÂAO}

Apesar de toda a evolução tecnológica alcançada pela oftalmologia nos últimos anos, ainda enfrentamos um grave problema de saúde pública que é a baixa de visão irreversível causada pela ambliopia.

Esta entidade chega a atingir $4 \%$ da população ${ }^{1}$, sendo o diagnóstico precoce, através dos exames de prevenção, a principal arma para seu tratamento.

Nos últimos anos os aparelhos de "Photoscreening" tem-se apresentado como um acessório muito útil nos exames populacionais em crianças.

Os "Photoscreening" têm como base de interpretação as características do reflexo retiniano da luz emitida por um flash acoplado a uma máquina fotográfica. O propósito do exame é identificar os principais fatores ambliopigênicos na população infantil, quais sejam: ametropias, anisometropias, estrabismos e cataratas.

A grande vantagem de sua aplicação é dispensar a informação verbal da criança, podendo portanto ser realizado na população com idade a partir de 6 meses de vida ${ }^{2}$ além de não necessitar de cicloplegia.

Atualmente os aparelhos evoluíram e se sofisticaram podendo ser encontrados conectados a computadores e aparelhos de interpretação automática $^{3}$. Neste artigo, estudaremos a sensibilidade, especificidade e precisão 
de uma máquina de "Photoscreening" na triagem de uma população infantil.

\section{MATERIAL E MÉTODOS}

Utilizamos a câmera portátil MTI PhotoScreener, produzida pela Medical Technology Innovations, que utiliza filmes polaroid preto e branco de alta velocidade(ISO 3200) produzindo na mesma foto duas imagens seqüenciais.

O exame é realizado a máquina a um metro de distância do paciente, em ambiente de penumbra, sem necessidade de cicloplegia. A máquina contém dispositivos de luz e som utilizados um para manter a fixação da criança e outro para verificar a distância correta de foco.

O aparelho dispara um flash que inicialmente faz uma varredura vertical de cima para baixo, seguido de outro disparo que faz a varredura horizontal da esquerda para direita.

Após o segundo disparo tem-se uma foto de revelação instantânea, contendo uma imagem na metade superior e outra na inferior.

A interpretação dos resultados se faz imediatamente após a revelação completa do filme ( \pm 2 minutos) pelas características de diâmetro pupilar, simetria do reflexo corneano, ausência de alguma opacidade de meios e presença de reflexos retinianos demonstrados como semiluas ou crescentes esbranquiçadas na área pupilar.

Semelhante à retinoscopia, se os reflexos possuírem concavidade voltada para cima e para esquerda são interpretados como miopia; concavidade para baixo e para direita, hipermetropia; diferenças de tamanho e direção das semiluas, em um mesmo olho, astigmatismo e finalmente, diferenças entre os reflexos dos dois olhos, anisometropia. Os resultados são qualitativos, não havendo portanto forma de mensurar as ametropias pelo estudo fotográfico (Figura 1).
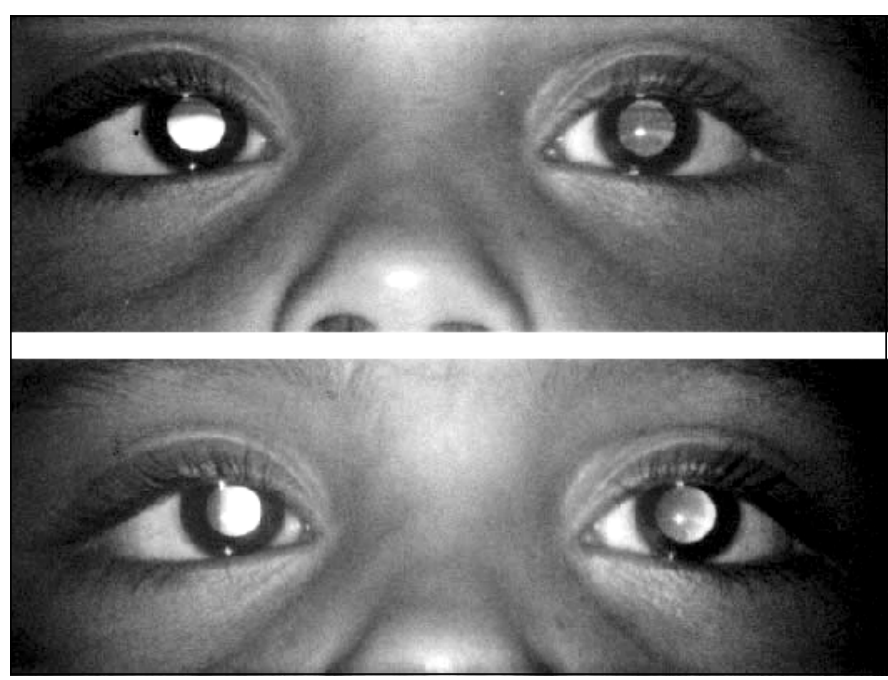

Fig. 1 - Paciente portador de Esotropia e Anisometropia Meniscos positivos $O D>O E$
Utilizamos os critérios fornecidos pelo fabricante para a interpretação das fotos (Tabela 1).

As fotos nas quais os diâmetros pupilares foram menores que $4 \mathrm{~mm}$ ou maiores que $8 \mathrm{~mm}$, ou onde havia erros de focalização ou fixação foram repetidas ou excluídas do trabalho.

Escolhemos aleatoriamente 109 crianças de um grupo de aproximadamente 800 , que estavam sendo examinadas para o projeto "Veja Bem Brasil". Após o "Photoscreening" elas foram submetidas aos exames de acuidade visual com e sem cicloplegia, alinhamento ocular, fundoscopia, biomicroscopia e retinoscopia, juntamente com as demais crianças. Os exames de "Photoscreening" e sua interpretação foram realizados por um grupo de profissionais distinto daquele que realizou os exames oftalmológicos.

As alterações oftalmológicas consideradas patológicas foram baseadas em conceitos da literatura pesquisada (Tabela 2) ${ }^{4,5}$.

Os dados do exame oftalmológico sob cicloplegia foram comparados à interpretação das fotos e o estudo de sensibilidade, especificidade, valores preditivos positivos e negativos foram analisados conforme a tabela 3 .

Foram analisados os exames de 109 crianças, sendo excluídas 2 fotos por serem consideradas tecnicamente inconclusivas. A amostra analisada ficou constituída 107 pacientes, sendo $55(51,4 \%)$ do sexo feminino e $52(48,59 \%)$ do masculino, com idade variando entre 6 e 15 anos (média 9,9 anos).

\section{Tabela 1. Critérios do fabricante}

- qualquer opacidade de meios

- qualquer assimetria do reflexo corneano (estrabismo)

- crescente miópica $\geq 2 \mathrm{~mm}$ em qualquer olho

- crescente hipermetrópica $\geq 3 \mathrm{~mm}$ em qualquer olho

- diferenças $\geq 2 \mathrm{~mm}$ entre as crescentes verticais e horizontaisno mesmo olho (astigmatismo).

Tabela 2. Critérios para identificação de patologias oculares

- qualquer alteração dos meios transparentes;

- estrabismo;

- miopias maiores que 1,00 D;

- hipermetropia maiores que 2,75 D;

- astigmatismos maiores que 1,00D;

- anisometropias maiores que 1,50D;

- anormalidades de polo posterior.

\section{Tabela 3. Estudo Estatístico}

$$
\begin{aligned}
& A=\text { verdadeiro positivo } \\
& B=\text { falso positivo } \\
& C=\text { falso negativo } \\
& D=\text { verdadeiro negativo } \\
& A+C=\text { total de doentes } \\
& B+D=\text { total de sadios }
\end{aligned}
$$

A) sensibilidade $=A /(A+C)$ (verdadeiro positivo/ total de doentes)

$B$ ) especificidade $=D /(B+D)$ (verdadeiro negativo/total de sadios)

C) valor preditivo positivo $=A /(A+B)$

D) valor preditivo negativo $=\mathrm{D} /(\mathrm{C}+\mathrm{D})$. 


\section{RESULTADOS}

As patologias encontradas nos 107 pacientes estão demonstradas na tabela 4 .

Encontramos no total $31(28,97 \%)$ pacientes com alguma patologia e $76(71,02 \%)$ dentro da normalidade, apresentando a seguinte análise de resultados:

Verdadeiro Positivo: 28; Falso negativo: 3; Total de Doentes: 31 .

Verdadeiro negativo: 73; Falso positivo: 3; Total de sadios: 76

Com base nos valores acima relatados o exame de "Photoscreening" apresentou: sensibilidade de $90,32 \%$, especificidade de $96,05 \%$, valores preditivos positivo e negativo de $90,32 \%$ e $96,05 \%$, respectivamente. Relação entre doentes e população estudada: $28,97 \%$.

O tempo médio de exame computando entrada da criança, realização da foto e observação dos critérios de aceitabilidade do resultado, foi de 2 minutos e 23 segundos. Foi necessária a repetição de 4 exames, no total dos 107 válidos.

\section{DISCUSSÃO}

Existem várias formas de se evidenciar a capacidade visual de uma criança, podendo ser divididas em exames subjetivos e objetivos.

Fazem parte do primeiro grupo, que depende da informação do paciente, os exames de acuidade visual pela leitura de optotipos e testes de estereopsia. O primeiro, apresenta como principal dificuldade só poder ser usado de forma confiável, em crianças maiores, já em fase verbal avançada e muitas vezes alfabetizadas. Nestas condições as chances de recuperação da ambliopia já diminuem significativamente. Os testes de estereopsia muitas vezes não são compreendidos pelas crianças mais novas, tornando seus resultados inconclusivos.

Os exames objetivos são realizados independente da informação verbal da população estudada, como por exemplo, o nistagmo optocinético, potencial visual evocado e auto-refrator e

\begin{tabular}{|lcc|}
\hline \multicolumn{3}{|c|}{ Tabela 4. Apresentação das patologias encontradas } \\
Miopia maior que 1,00D & $\mathbf{N}^{\circ}$ de casos & $\%$ \\
Hipermetropia maior que 2,75D & 8 & 19,0 \\
Astigmatismo maior que 1,00D & 12 & 28,5 \\
Anisometropia maior que 1,50D & 9 & 21,4 \\
Esotropia & 6 & 14,2 \\
Hipertropia & 2 & 4,7 \\
Lesão de córnea & 1 & 2,3 \\
Luxação de cristalino & 1 & 2,3 \\
Pseudofacia & 2 & 4,7 \\
TOTAL & 1 & 2,3 \\
OBS: os casos onde havia astigmatismo miópico ou hipermetrópico com o \\
componente esférico maior que o padrão de normalidade clínica, foram \\
computados nas duas patologias. Utilizou-se o critério de equivalente esférico \\
para mensurar as ametropias.
\end{tabular}

os "Photoscreening". Se por um lado estes testes permitem o diagnóstico mais precoce, por não exigirem a informação verbal, são na sua maioria mais dispendiosos, tornando muitas vezes impraticável sua utilização na seleção de grandes populações. Como exceção citamos os exames ortóptico e de supressão, além do exame do reflexo vermelho e retinoscopia a distância.

Durante este trabalho pudemos observar a validade do "Photoscreening" no estudo da população infantil por apresentar resultados confiáveis nos critérios de sensibilidade, especificidade e preditivos, comparados a outros métodos de estudo populacional, na faixa etária estudada ${ }^{6}$.

A literatura levantada demonstra uma diminuição nos valores de sensibilidade e especificidade à medida que a faixa etária estudada diminui, principalmente nas crianças pré-verbais ${ }^{7}$.

É um exame de fácil execução, apresentando curva de aprendizado rápida, podendo portanto ser feito por técnicos, paramédicos ou professores. $\mathrm{O}$ aparelho utilizado apresenta grande vantagem em ser portátil e emitir uma foto instantânea que proporciona análise imediata do resultado, podendo, se necessário, repetí-la antes do paciente ser dispensado, além de se ter um registro do exame, permitindo discutí-lo em caso de dúvida. Por sua rápida execução (tempo médio de 2 minutos e 23 segundos), consegue-se estudar um grande grupo em pouco tempo.

Quando se trata de estudo populacional um fator importante a ser lembrado é o custo dos exames. Neste caso a máquina custou 3000 dólares e as fotos em torno de 2 dólares cada, se observando uma diluição do custo a medida que o número de pacientes examinados aumenta.

O cuidado maior deve ser tomado durante a análise das fotos, onde pequenos detalhes como tamanho das crescentes, diâmetro pupilar pequeno e alinhamento do reflexo corneano podem gerar interpretações equivocadas e diagnósticos imprecisos. Requerendo portanto experiência do examinador.

Quando analisamos os 3 casos de falso negativo, constatamos que em 1 deles o "Photoscreening" foi considerado como normal e sob cicloplegia foi encontrado miopia de $-1,50 \mathrm{D}$, em cada olho. Como se tratava de criança melanoderma e com íris muito pigmentada, existe a possibilidade da cicloplegia ter sido insatisfatória. Em outro caso, a criança apresentava esotropia, que foi diagnosticada corretamente, porém ao exame da foto não se constatou hipermetropia de 3,00D no olho fixador. No último paciente, houve erro pelo não diagnóstico da anisometropia hipermetrópica do olho esquerdo.

No estudo dos 3 falso-positivos houve divergência entre os valores das crescentes que demonstravam ametropias maiores do que as encontradas sob cicloplegia, sendo dois casos de hipermetropia e um de astigmatismo.

Outro dado que chamou atenção foi a elevada incidência da população com alguma patologia $(28,97 \%)$, que talvez se justifique, visto que, parte das crianças que foram examinadas naquele dia pelo projeto "Veja Bem Brasil" já haviam sido previamente selecionadas, em outras escolas.

Ao final, podemos concluir que o exame de "Photoscree- 
ning" se mostrou confiável, para triagem de crianças na idade escolar, apresentando vantagens na como a rapidez e facilidade de sua realização, bem como dispensar o uso de cicloplégicos.

\section{SUMMARY}

Purpose: To study the accuracy of the MTI Photoscreener in the screening of an infantile population, for amblyopia causing factors such as ametropias, anisometropias, strabismus, congenital cataracts and opacities of the media. Methods: During the execution of a study project "Veja Bem Brasil" ("See Well Brazil"), the AA used the MTI Photoscreener to examine 107 children, with ages varying from 6 to 15 years, and compared these results with those of a following complete ophthalmic examination.

Results: The end results gave us 31 (28.97\%) children with an ophthalmic pathology and 76 (71.02\%) within normal limits. Statistical analysis showed the following values: sensivitity, 90.32\%; specificity, $96.05 \%$; positive predictive values and negative predictive values of $90.32 \%$ e $96.05 \%$, respectively.
Conclusion: The above results showed the "photoscreening" test to be reliable to screen young patients of school age.

Keywords: Screening; Photoscreening (children); Amblyopia.

\section{REFERÊNCIAS BIBLIOGRÁFICAS}

1. Bicas HEA, Brik M et al. Estado Atual do Tratamento da Ambliopia. Arq Bras Oftalm 1987;50(1):5-12.

2. Atiknson J, Braddick OJ et al. Screening for refractive errors in six to nine month old infants by photorefraction. Brit J Ophthalmol 1984;68:105-12.

3. Kurt S. Preschool Vision Screening: Rationale, Methodology and outcome. Survey Ophthalmol 1996;41:3-30.

4. Fredman HL, Preston KL. Polaroid "Photoscreening" for Amblyogenic Factors - An improved Methodology. Ophthalmology 1992;99:18-28.

5. Ottar WL, Scott WE, Holgado SI. "Photoscreening" for an Amblyogenic Factors. J Pediatr Ophthalmol Strabismus 1995;32:289-95.

6. Brik D, Moreira JBC et al. Retinoscopia a dois metros na detecção de fatores Causadores de Ambliopia em crianças de Curitiba. Arq Bras Oftalm 1995;58(1):5-9.

7. Hatch SW, Tibbles CD et al. Validity and Reliability of The MTI Photoscreener. Optom Vis Sci 1997;74(10):859-64.

\section{5 ' SIMPÓSIO INTERNACIONAL DE UVEÍTES}

\section{6 a 28 de março de 2.000 - Buenos Aires - Argentina}

\section{Simpósio organizado pelo Grupo Internacional de Estudos em Uveítes (IUSG)}

Contará com a presença de 50 especialistas em uveítes do mundo inteiro.

Os membros da Sociedade Brasileira de Uveítes e do Conselho Brasileiro de Oftalmologia terão destaque especial no congresso.

\section{INFORMAÇÕES:}

No Brasil: Dr. Rubens Belfort Jr.

R. Botucatu, 822 - CEP 04023-062 - São Paulo - SP

Fax: (55-11) 573-4002
Na Argentina: Dr. Cristóbal Couto

Fax: (54-1) 953-2092 